This is a preprint of: "Limit cycles in 4-star-symmetric planar piecewise linear systems", Claudio A. Buzzi, Joao Carlos Medrado, Joan Torregrosa, J. Differential Equations, vol. 268(5), 2414$2434,2020$.

DOI: [https://doi.org/10.1016/j.jde.2019.09.008]

\title{
LIMIT CYCLES IN 4-STAR-SYMMETRIC PLANAR PIECEWISE LINEAR SYSTEMS
}

\author{
CLAUDIO A. BUZZI, JOAO C. MEDRADO, AND JOAN TORREGROSA
}

\begin{abstract}
Our interest is centered in the study of the number of limit cycles for nonsmooth piecewise linear vector fields on the plane when the switching curve is $x y=0$. We consider the symmetric case. That is, one vector field defined in the odd quadrants and the other in the even ones. We deal with equilibrium points of center-focus type, with matrices in real Jordan form, in each vector field when the infinity is monodromic. In this case, we provide the center classification at infinity, we prove that the maximum order of a weak focus is five. Moreover, we show the existence of systems exhibiting five limit cycles bifurcating from infinity.
\end{abstract}

\section{INTRODUCTION}

These last years a big interest appeared for studying nonsmooth piecewise differential systems, motivated mainly by their biological and engineering applications. In particular, piecewise linear differential systems have been used to model many real processes and different modern devices, for more details see for instance $[1,11]$ and the references therein.

In piecewise-smooth dynamical systems, the phase space is divided in regions with different smooth vector fields in each one. At the boundary between smooth regions, the vector field could be neither continuous nor differentiable. But it can be defined using the Filippov convention, see [14]. Such systems arise in several applications, and their analysis constitutes an exciting field of current research. In fact, this nonsmoothness may cause a whole new rich family of bifurcations that do not appear in smooth systems. Due to the nondifferentiability, the analysis of such bifurcations prevents the use of standard local methods (see for example [2]), and thus demands the development of new techniques.

In general, many works consider the piecewise smooth vector fields when the switching manifold (or discontinuity manifold), $\Sigma=f^{-1}(p)$, is a submanifold given by the preimage of a regular value, $p$, of a differentiable real function. Although there are few works considering the case when the switching manifold has a singularity (it is the preimage of a nonregular value of a function), we can found some real phenomena modeled by this type of systems. Different planar models with $f(x, y)=$ $x y$ can be found in the literature. For example, a model of a steam-engine ([3]), a periodically forced double-belt friction oscillator ([13]), or a two-mass model of the human vocal folds used to simulate their oscillatory motion at phonation ([22]). There are models in higher dimensions, $\left(x_{1}, x_{2}, \ldots, x_{n}\right)$, where the nonlinearities depend also on $\left|x_{i}\right|$, for $i=1, \ldots, n$. These piecewise differential equations are defined with discontinuity varieties having also a nonregular intersection, that is

2010 Mathematics Subject Classification. Primary: 34C25, 34C07, 37C27.

Key words and phrases. Non-smooth differential system, 4-cross-symmetric, cyclicity, limit cycles, center-focus problem. 
$x_{1} x_{2} \cdots x_{n}=0$. For example, among other bifurcations, the study of periodic orbits by a Hopf bifurcation in a model for ship maneuvering is studied in [4].

An interesting phenomenon that appears in the analysis of the models described by (smooth or not) differential equations, is the existence of periodic behaviors. In particular the study of the number, stability, location,... of isolated periodic orbits. When we restrict to planar polynomial vector fields the problem of finding the maximum number of limit cycles remains open. It is part of the famous 16thHilbert problem. This problem make no sense for linear vector fields because they have no limit cycles. But as there exist limit cycles crossing $\Sigma$ for piecewise linear vector fields we can consider the generalized 16th-Hilbert problem. We call Hilbert number to this maximum when it exists. The Hilbert number for piecewise linear vector fields defined in two zones separated by a straight line is only known for some particular cases. For the continuous case, this number was conjectured to be one by Lum and Chua in [23] and proved by Freire et al. in [15]. For the discontinuous case without sliding motion, see [14], in [24] was proved that the Hilbert number is also one. When there is sliding motion, the best known results show that the Hilbert number is bigger or equal than three (see $[6,16,17,21]$ ). In this paper we consider the class of 4-star-symmetric planar piecewise linear systems. That is, when $\Sigma=\{(x, y): x y=0\}$ and there are only two piecewise linear systems defined in the odd and even quadrants, respectively. Our main result shows that the Hilbert number in this class is at least five. The same lower bound is also obtained in [7] studying the case having one linear system defined in the first quadrant and another in the other quadrants.

We deal with piecewise linear systems of the form

$$
\left\{\begin{array}{l}
x^{\prime}=\alpha^{ \pm} x-\beta^{ \pm} y+\gamma^{ \pm}, \\
y^{\prime}=\beta^{ \pm} x+\alpha^{ \pm} y+\delta^{ \pm},
\end{array}\right.
$$

defined in $\Sigma^{ \pm}=\left\{(x, y) \in \mathbb{R}^{2}: \pm x y>0\right\}$ when $\beta^{ \pm} \neq 0$. On $\Sigma=\left\{(x, y) \in \mathbb{R}^{2}: x y=\right.$ $0\} \backslash\{(0,0)\}$ we use the Filippov convention, see [14]. As we are interested in periodic motions, we will study only the case $\beta^{+} \beta^{-}>0$. By means of a time-rescaling, if necessary, and defining $a^{ \pm}=\alpha^{ \pm} / \beta^{ \pm}, b^{ \pm}=\gamma^{ \pm} / \beta^{ \pm}$, and $c^{ \pm}=\delta^{ \pm} / \beta^{ \pm}$, system (1) becomes

$$
\left\{\begin{array}{l}
x^{\prime}=a^{ \pm} x-y+b^{ \pm} \\
y^{\prime}=x+a^{ \pm} y+c^{ \pm}
\end{array}\right.
$$

It is not restrictive, doing the change $(x, y, t) \mapsto(-x, y,-t)$ if necessary, to assume also that $a^{+}>0$. See Figure 1 .

When $b^{ \pm}=c^{ \pm}=0$ the origin is the unique equilibrium point, which is of centerfocus type, for each linear system in (2) defined in $\Sigma^{ \pm}$, respectively. Consequently, the origin is an equilibrium point of monodromic type for system (2). Its stability is given by the sign of $a^{+}+a^{-}$, see [28]. That is, stable (unstable) when it is negative (positive). Moreover, the Poincaré return map is globally linear and the origin is a global focus $\left(a^{+}+a^{-} \neq 0\right)$ or a global center $\left(a^{+}=-a^{-}\right)$. In this case, we have no limit cycles. Otherwise, some sliding motion can appear near the origin of the vector field (2), in particular the monodromy property could fail. But this property is kept near the infinity when we extend (2) to the Poincaré disk, see [12]. In Figure 1, the solutions are depicted far from the origin. We present results about the existence of limit cycles and its maximum number for system (2) near the infinity. In this neighborhood all the trajectories are of crossing type. This is done by an adequate 


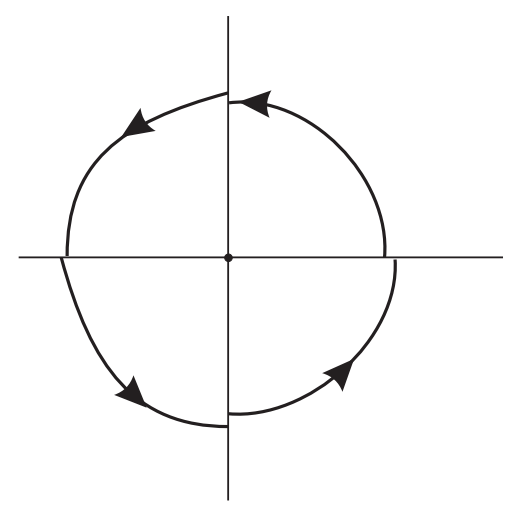

FiguRE 1. The phase portrait of system (2) near infinity.

polar coordinates change, $(x, y)=\left(R^{-1} \cos \theta, R^{-1} \sin \theta\right)$, that moves the infinity to the origin. After this change, this problem is related with the classical center-focus problem. More concretely, we study the series expansion of the return map near the origin and we compute the Lyapunov quantities. More details about this theory for smooth systems can be seen in [2]. Our computations deal near a focus-focus case. The first stability condition for a general system with an equilibrium point at the origin of focus type and defined in the regions depicted in Figure 1 was done in $[19,28]$. Previously, but when the discontinuity curve is a straight line, the first terms of the series expansion were obtained also for the fold-fold and fold-focus cases in [10]. The approach that we will use is closer to the classical smooth one. For a general introduction to the center-focus problem for smooth systems see [26]. In this paper we extend this theory to be applied in our class of piecewise vector fields.

Next, we present the main results of this work. In the first we provide a lower bound for the maximum number of isolated periodic orbits (limit cycles) for system (2) near the infinity. In the second we classify the centers at infinity. We observe that the listed families are not disjoint.

Theorem 1.1. There exist values of the parameters such that system (2) has five limit cycles bifurcating from infinity.

Theorem 1.2. System (2) has a center at infinity if and only if the parameters belong to one of the next families:

$$
\begin{aligned}
\mathcal{F}_{1}= & \left\{b^{-}+b^{+}=c^{-}-c^{+}=0\right\}, \\
\mathcal{F}_{2}= & \left\{(A a-1) b^{-}-(A+a) c^{-}=(A a-1) b^{+}+(A+a) c^{+}=0\right\}, \\
\mathcal{F}_{3}= & \left\{b^{-}-b^{+}=c^{-}+c^{+}=0\right\}, \\
\mathcal{F}_{4}^{ \pm}= & \left\{(A \pm a) b^{-}-(A \mp a) b^{+}=(A \pm a) c^{-}-(A a \pm 1) b^{+}=\right. \\
& \left.(A \pm a) c^{+}+(A a \mp 1) b^{+}=0\right\}, \\
\mathcal{C}_{1}= & \left\{a=b^{-}-b^{+}=c^{-}-c^{+}=0\right\}, \\
\mathcal{C}_{2}= & \left\{a=b^{-}-c^{-}=b^{+}+c^{+}=0\right\}, \\
\mathcal{C}_{3}^{ \pm}= & \left\{a=b^{-} \pm c^{-}=b^{+} \pm c^{+}=0\right\},
\end{aligned}
$$

where $a=a^{+}=-a^{-}$and $A=\mathrm{e}^{-a \frac{\pi}{2}}$. 
This paper is structured as follows. In Section 2 we introduce the technical results to define, via the coefficients of the series expansion of the solution near the origin, the center conditions (Lyapunov quantities). They are used, in Section 3, to classify the centers for proving Theorem 1.2. The study of the isolated periodic orbits is done in Section 4 with a generalization of the classical degenerate Hopf bifurcation. We study when the infinity is a weak focus and which is its maximal order. Besides the proof of Theorem 1.1 we prove that there are no weak foci of order greater than five. The five limit cycles appear proving the existence of a weak focus of order five, via Poincaré-Miranda's Theorem ([20]), together with a generic unfolding, using the Implicit Function Theorem, in family (2). To prove the existence of a generic unfolding we closely follow the approach used in $[8,9,18]$. As this problem has only six parameters $\left(a^{ \pm}, b^{ \pm}, c^{ \pm}\right)$, it is quite natural that the number of limit cycles be five. We notice that the difficulties for proving the existence of such limit cycles are devoted to the fact that our perturbation should be restricted to be inside the considered family. When the perturbation is analytic the explicit computations are not necessary to be done. See, for example, [5]. The computations are done with the Computer Algebra System MAPLE and with a rigorous rational interval analysis.

\section{Canonical form and the Poincaré Return map}

As we are interested in the periodic orbits bifurcating from infinity, by the monodromy property, the natural coordinates to study the problem are

$$
(x, y)=\left(R^{-1} \cos \theta, R^{-1} \sin \theta\right) .
$$

Then, a neighborhood of infinity of (2) is moved to a neighborhood of the origin of equation

$$
R^{\prime}=\frac{d R}{d \theta}=f(R, \theta)=\frac{-a R-(b \cos \theta+c \sin \theta) R^{2}}{1+(c \cos \theta-b \sin \theta) R}
$$

where $(a, b, c)=\left(a^{+}, b^{+}, c^{+}\right)$if $\theta \in(0, \pi / 2) \cup(\pi, 3 \pi / 2)$ and $(a, b, c)=\left(a^{-}, b^{-}, c^{-}\right)$if $\theta \in(\pi / 2, \pi) \cup(3 \pi / 2,2 \pi)$. Clearly, for $R$ small enough the solution is well defined for $\theta \in[0,2 \pi]$.

We consider, for each $i=1,2,3,4$, and $\rho$ small enough, the solution $R_{i}(\theta, \rho)$ of (4) satisfying the initial condition $R_{i}((i-1) \pi / 2, \rho)=\rho$. Then we define the first return map on each quadrant $i=1,2,3,4$ as $\Pi_{i}(r)=R_{i}(i \pi / 2, \rho)$ and the total return map as the ordered composition of all of them, $\Pi(\rho)=\Pi_{4}\left(\Pi_{3}\left(\Pi_{2}\left(\Pi_{1}(\rho)\right)\right)\right)$. We observe that $\Pi$ is analytic, in a neighborhood of the origin, because it is defined by composition of analytic maps. Consequently, the periodic orbits of (2) are the fixed points of $\Pi$ or, alternatively, the zeros of a displacement function $\Delta(\rho)=$ $\left(\Pi_{2} \circ \Pi_{1}\right)(\rho)-\left(\Pi_{3}^{-1} \circ \Pi_{4}^{-1}\right)(\rho)$.

Next result relates the first return maps in each quadrant with the defined in the first one.

Lemma 2.1. Let $R(\theta, \rho)$ be the solution of (4) satisfying the initial condition $R(0, \rho)=\rho$, then we write $\Pi_{(a, b, c)}(\rho)=R(\pi / 2, \rho)$. Then, $\Pi_{1}(\rho)=\Pi_{\left(a^{+}, b^{+}, c^{+}\right)}(\rho)$, $\Pi_{2}(\rho)=\Pi_{\left(a^{-}, c^{-},-b^{-}\right)}(\rho), \Pi_{3}(\rho)=\Pi_{\left(a^{+},-b^{+},-c^{+}\right)}(\rho)$, and $\Pi_{4}(\rho)=\Pi_{\left(a^{-},-c^{-}, b^{-}\right)}(\rho)$.

The proof follows doing an adequate translation in the angle variable that moves the respective quadrant to the first one. From this lemma we only need the expression of $\Pi_{(a, b, c)}(\rho)$. The series expansion in $\rho$ can be obtained from the series expansion of the solution $R(\theta, \rho)$ of (4) with $R(0, \rho)=\rho$. That is, substituting $R(\theta, \rho)=$ 
$\sum_{i=1}^{\infty} R_{i}(\theta) \rho^{i}$, with $R_{1}(0)=1$ and $R_{i}(0)=0$, for $i=2, \ldots$, in (4). Straightforward computations show that $R_{1}(\theta)=\mathrm{e}^{-a \theta}$ and $R_{2}(\theta)=\mathrm{e}^{-2 a \theta}(b \sin \theta-c \cos \theta)+\mathrm{e}^{-a \theta} c$. We do not show the other terms because of the size of them. From the previous definition we can write

$$
\begin{aligned}
\Pi_{(a, b, c)}(\rho)= & R(\pi / 2, \rho)=\mathrm{e}^{-a \frac{\pi}{2}} \rho+\left(\frac{a^{2} c-2 a b-c}{a^{2}+1} \mathrm{e}^{-a \frac{\pi}{2}}+\frac{a^{2} b+2 a c-b}{a^{2}+1} \mathrm{e}^{-a \pi}\right) \rho^{2} \\
& +\left(\frac{a^{4} c^{2}-6 a^{3} b c+7 a^{2} b^{2}-5 a^{2} c^{2}+10 a b c-b^{2}+2 c^{2}}{2\left(a^{2}+1\right)} \mathrm{e}^{-a \frac{\pi}{2}}\right. \\
& +\frac{2\left(a^{2} c-2 a b-c\right)\left(a^{2} b+2 a c-b\right)}{a^{2}+1} \mathrm{e}^{-a \pi} \\
& \left.+\frac{3 a^{4} b^{2}+10 a^{3} b c-3 a^{2} b^{2}+9 a^{2} c^{2}-6 a b c+2 b^{2}+c^{2}}{2\left(a^{2}+1\right)} \mathrm{e}^{-3 a \frac{\pi}{2}}\right) \rho^{3}+O\left(\rho^{4}\right) .
\end{aligned}
$$

Using Lemma 2.1 we can get the first terms of the series development of the complete Poincaré map $\Pi(\rho)$, or equivalently, the displacement function $\Delta(\rho)=$ $\sum_{i=1}^{\infty} \hat{R}_{i} \rho^{i}$, with

$$
\begin{aligned}
\hat{R}_{1}= & \mathrm{e}^{-\left(a^{+}+a^{-}\right) \frac{\pi}{2}}-\mathrm{e}^{\left(a^{+}+a^{-}\right) \frac{\pi}{2}} \\
\hat{R}_{2}= & \frac{\left(a^{-}\right)^{2} c^{-}-2 a^{-} b^{-}-c^{-}}{\left(a^{-}\right)^{2}+1}\left(\mathrm{e}^{-\left(a^{-}+a^{+}\right) \pi}-\mathrm{e}^{\left(a^{-}+a^{+}\right) \frac{\pi}{2}}\right) \\
& +\frac{\left(a^{+}\right)^{2} c^{+}-2 a^{+} b^{+}-c^{+}}{\left(a^{+}\right)^{2}+1}\left(\mathrm{e}^{-\left(a^{-}+a^{+}\right) \frac{\pi}{2}}-\mathrm{e}^{\left(a^{-}+a^{+}\right) \pi}\right) \\
& -\frac{1}{\left(\left(a^{+}\right)^{2}+1\right)\left(\left(a^{-}\right)^{2}+1\right)}\left(\left(\left(a^{-}\right)^{2}\left(a^{+}\right)^{2}-1\right)\left(b^{-}-b^{+}\right)\right. \\
& +\left(\left(a^{-}\right)^{2}-\left(a^{+}\right)^{2}\right)\left(b^{-}+b^{+}\right)+2 a^{-} a^{+}\left(a^{+} c^{-}-a^{-} c^{+}\right) \\
& \left.+2\left(a^{-} c^{-}-a^{+} c^{+}\right)\right)\left(\mathrm{e}^{-\left(a^{-}+2 a^{+}\right) \frac{\pi}{2}}-\mathrm{e}^{\left(2 a^{-}+a^{+}\right) \frac{\pi}{2}}\right) .
\end{aligned}
$$

The stability of the origin for system (4) is given by the sign of the first nonvanishing coefficient $\hat{R}_{i}$. The next proposition is equivalent to the classical result for the centerfocus problem in the smooth scenario.

Proposition 2.2. The first non-vanishing term $\hat{R}_{i}$ corresponds to an odd index $i$.

Proof. The proof is the same as the one provided in [2, Chap. IX, Sect. 24] for the smooth context. Because, it uses only the fact the function $f$, in (4), satisfies the symmetry relation $f(-R, \theta+\pi)=-f(R, \theta)$.

We notice that, in the general case with four quadrants without symmetry, the above result is not valid.

It is natural to define the first nonzero value when the previous ones are zero. Then, using the above result, we define the Lyapunov quantity of order $i$ of the origin of system (4) as

$$
L_{0}=\hat{R}_{1}=\mathrm{e}^{-\left(a^{+}+a^{-}\right) \frac{\pi}{2}}-\mathrm{e}^{\left(a^{+}+a^{-}\right) \frac{\pi}{2}}
$$

or

$$
L_{i}=\left.\hat{R}_{2 i+1}\right|_{\left\{L_{1}=\cdots=L_{i-1}=0\right\}}, \text { for } i=1,2, \ldots
$$


So, as $L_{0}=0$ if and only if $a^{+}+a^{-}=0$, straightforward computations show that

$$
\begin{aligned}
L_{1}= & \frac{\left(a^{+} c^{-}-a^{+} c^{+}+b^{-}+b^{+}\right)\left(a^{+} c^{-}+a^{+} c^{+}+b^{-}-b^{+}\right)}{\left(a^{+}\right)^{2}+1} \\
& -\frac{\left(a^{+} b^{-}+a^{+} b^{+}-c^{-}+c^{+}\right)\left(a^{+} b^{-}-a^{+} b^{+}-c^{-}-c^{+}\right)}{\left(a^{+}\right)^{2}+1} \mathrm{e}^{-\pi a^{+}} .
\end{aligned}
$$

Although in the next section we will need the explicit expression of the first five Lyapunov quantities, we do not present all of them because of their size.

This symmetric case reproduces the same properties as the smooth scenario. Then we say that the origin of (4) is a hyperbolic focus when $L_{0} \neq 0$. When $L_{0}=L_{1}=$ $\cdots=L_{i-1}=0$ and $L_{i} \neq 0$ the origin of (4) is a weak focus of order $i$. In fact, the case $L_{0}=0$ corresponds, for an analytic vector field, to a monodromic equilibrium point with a Jacobian matrix having trace equal to zero and positive determinant. We will say that the origin is an equilibrium point of (4) of center-focus type. The next result corresponds to the bifurcation of a limit cycle from the origin by a Hopf bifurcation when we perturb a system having a weak focus of order 1 .

Proposition 2.3. Let us consider equation (4) with $a^{+}+a^{-}=\varepsilon$ and when $\varepsilon=0$ we assume that $L_{1}<0(>0)$ in (6). Then, for $\varepsilon=0$ the origin is a stable (unstable) weak focus of order 1 of (4). Moreover, for $\varepsilon<0(>0)$ small enough, a stable (unstable) limit cycle bifurcates from the origin.

The degenerate Hopf bifurcation, obtaining $k$ limit cycles from a weak focus of order $k$, can be also studied similarly as in the classical theory of analytic systems. See $[2,27]$.

\section{The CENTER PROBLEM}

The studies of the necessary and sufficient conditions in order that system (4) has a center at the origin or system (2) has a center at infinity are equivalent, due to the change of coordinates introduced in previous section. The proof of Theorem 1.2 follows from the results of this section. Proposition 3.2 proves the necessary conditions and Proposition 3.3 proves the sufficiency of them.

From the results of the above section we can assume $L_{0}=0$, that is $a^{+}=-a^{-}=a$, see (5). From the expression $L_{1}$ in (6) we will introduce a change of variables in the parameter space, because the size of the expressions of the Lyapunov quantities decrease. This is done in the next lemma, which provides the structure of the first five Lyapunov quantities for system (4).

Lemma 3.1. Consider system (4) with $a^{+}=a, a^{-}=-a, A=\mathrm{e}^{-a \frac{\pi}{2}}$ and

$$
\begin{array}{ll}
B_{1}=a\left(b^{-}+b^{+}\right)-\left(c^{-}-c^{+}\right), & C_{1}=a\left(c^{-}-c^{+}\right)+b^{-}+b^{+}, \\
B_{2}=a\left(b^{-}-b^{+}\right)-\left(c^{-}+c^{+}\right), & C_{2}=a\left(c^{-}+c^{+}\right)+b^{-}-b^{+} .
\end{array}
$$

Then, the origin is an equilibrium point of center-focus type of (4), $L_{0}=0$, and the first five Lyapunov quantities write as

$$
L_{k}=\frac{M_{k}\left(a, A, B_{1}, B_{2}, C_{1}, C_{2}\right)}{\left(a^{2}+1\right)^{2 k-1}}
$$

for some polynomials $M_{k}$ with rational coefficients of degree $6 k-2$. Moreover, they are also homogeneous polynomials of degree $2 k$ in the variables $\left(B_{1}, B_{2}, C_{1}, C_{2}\right)$. 
More concretely,

$$
\begin{aligned}
M_{1}= & -\left(A^{2} B_{1} B_{2}-C_{1} C_{2}\right), \\
M_{2}= & \left(a A^{3}\left(3 A^{2}-8 a A+9\right) B_{1}^{3} B_{2}-A^{3}(4 a A-3) B_{1}^{3} C_{2}\right. \\
& +3 A^{2}\left(3 a^{2} A-A-4 a\right) B_{1}^{2} B_{2} C_{1}+a A^{3}\left(3 A^{2}+1\right) B_{1} B_{2}^{3} \\
& -3 A^{3}\left(4 a A+3 a^{2}-1\right) B_{1} B_{2}^{2} C_{2}+a A\left(A^{2}+3\right) B_{1} B_{2} C_{1}^{2} \\
& +a A\left(9 A^{2}+8 a A+3\right) B_{1} B_{2} C_{2}^{2}-(3 A+4 a) B_{1} C_{2}^{3} \\
& \left.+a^{2} A^{3} B_{2}^{3} C_{1}-a^{2} A B_{2} C_{1}^{3}\right) / 12 .
\end{aligned}
$$

We have not written all the polynomials $M_{k}$ because of their size. In particular, $M_{3}, M_{4}$, and $M_{5}$ have 303,1175 , and 3398 monomials, respectively.

Proposition 3.2. Let $a^{+}=a, a^{-}=-a, A=\mathrm{e}^{-a \frac{\pi}{2}}$ and $B_{1}, B_{2}, C_{1}$, and $C_{2}$ defined in (7). The conditions

$$
\begin{array}{lll}
\mathcal{F}_{1}=\left\{B_{1}=C_{1}=0\right\}, & \mathcal{C}_{1}=\left\{a=B_{1}=C_{2}=0\right\}, \\
\mathcal{F}_{2}=\left\{C_{1}=A B_{1}, C_{2}=A B_{2}\right\}, & \mathcal{C}_{2}=\left\{a=0, B_{1}=-C_{1}, B_{2}=-C_{2}\right\}, \\
\mathcal{F}_{3}=\left\{B_{2}=C_{2}=0\right\}, & \mathcal{C}_{3}^{ \pm}=\left\{a=0, B_{1}= \pm C_{2}, B_{2}= \pm C_{1}\right\}, \\
\mathcal{F}_{4}^{ \pm}=\left\{B_{1}=C_{2}=0, C_{1}= \pm A B_{2}\right\}, &
\end{array}
$$

are necessary for system (4) has a center at the origin.

We notice that the families defined in the above result are exactly the ones appearing in Theorem 1.2.

Proof of Proposition 3.2. The proof follows studying when all the Lyapunov quantities that appear in Lemma 3.1 vanish. First we consider the condition $B_{2}=0$ and then $B_{2} \neq 0$.

We start with the condition $B_{2}=0$. From the expression of $M_{1}$ in (9) it is clear that if $L_{1}=0$ then $C_{2}=0$ or $C_{1}=0$. The first case is in $\mathcal{F}_{3}$. For the second case, that is $C_{1}=0$ and $C_{2} \neq 0$, computing the next Lyapunov quantities, see (8), we obtain $M_{k}=B_{1} C_{2} P_{k}\left(a, A, B_{1}, C_{2}\right)$, for $k=2,3,4,5$, with $P_{k}$ a polynomial with rational coefficients of degree $6 k-5$. Clearly, $B_{1}=0$ vanish all the quantities and defines family $\mathcal{F}_{1}$. Only remains the study of vanishing all the polynomials $P_{k}$. Computing the crossed resultants of $P_{k}$ with $P_{2}$ we obtain $R_{2, k}=a^{2} A^{8}\left(A^{2}+1\right)^{2} C_{2}^{4 k-4} Q_{k}(a, A)^{2}$, for $k=3,4,5$, with $Q_{k}$ polynomials with rational coefficients of degrees 6,13 , and 20 , respectively. When $a=0$ all $R_{2, k}$ vanish identically and we obtain $\mathcal{C}_{3}^{ \pm}$. The proof of the case $B_{2}=0$ finishes showing that, when $a \neq 0, B_{1} \neq 0, C_{2} \neq 0$, and $C_{1}=0$, the system has a weak focus of order 4 or 3 , depending if $Q_{3}(a, A)$ is zero or not. The existence of a focus of order 4 implies that $Q_{4}$ is not zero when $Q_{3}$ is. This is because, if the curves $Q_{3}(a, A)=0$ and $Q_{4}(a, A)=0$ have an intersection point $\left(a^{*}, A^{*}\right), a^{*}$ will be a root of the resultant of $Q_{3}$ and $Q_{4}$ with respect to $A$, which is a polynomial with rational coefficients of degree 26 in $a^{2}$. Similarly, $A^{*}$ will be a root of another polynomial, also with rational coefficients, of degree 27 in $A^{2}$. Consequently, both $a^{*}$ and $A^{*}$ will be algebraic numbers and, from the definition of $A$, we have that $\left(A^{*}\right)^{2}=\mathrm{e}^{-\pi a^{*}}$. This last assertion contradicts Gelfond-Scheneider Theorem, see [25, Pag. 134], which says that "If $\alpha$ and $\beta$ are algebraic number with $\alpha \neq 0, \alpha \neq 1$, and $\beta \notin \mathbb{Q}$ then $\alpha^{\beta}$ is transcendental." Because, as $\mathrm{e}^{\pi}=i^{-2 i}$, we will have $\left(A^{*}\right)^{2}=\mathrm{e}^{-\pi a^{*}}=i^{2 i a^{*}}$ is transcendental but it is algebraic. 
We continue assuming now that $B_{2} \neq 0$. From the expression of $M_{1}$ in (9) we have $B_{1}=C_{1} C_{2} /\left(A^{2} B_{2}\right)$, then the next Lyapunov quantities, by (8), write

$$
M_{k}=C_{1}\left(C_{2}-A B_{2}\right) B_{2}^{1-2 k} A^{5-4 k} P_{k}\left(a, A, B_{2}, C_{1}, C_{2}\right),
$$

for $k=2,3,4,5$, with each $P_{k}$ is a polynomial with rational coefficients of degree $12(k-1)$. Consequently, there are two simple cases, $C_{1}=0$ and $C_{2}=A B_{2}$, that define families $\mathcal{F}_{1}$ and $\mathcal{F}_{2}$, respectively. Only remains the study of vanishing all the polynomials $P_{k}$ when $B_{2} \neq 0, C_{1} \neq 0$, and $C_{2} \neq A B_{2}$.

The intersection points of the varieties $P_{k}=0$ can be studied also from the two by two crossed resultants. Straightforward computations show that

$$
\operatorname{res}\left(P_{2}, P_{k}, C_{1}\right)=a^{2} A^{2 k}\left(A^{2}+1\right)^{2} B_{2}^{4 k-4} C_{2}^{2}\left(A B_{2}-C_{2}\right)^{4} R_{2, k}\left(a, A, B_{2}, C_{2}\right),
$$

for $k=3,4,5$, for some $R_{2, k}$ polynomials with rational coefficients of degree $17 k-27$. Here we have also two simple cases, $a=0$ or $C_{2}=0$. In the first case, $P_{k}=$ $C_{2}^{2}\left(B_{2}+C_{2}\right)\left(B_{2}^{2}-C_{1}^{2}\right) Q_{k}\left(B_{2}, C_{1}, C_{2}\right)$, for $k=2,3,4,5$, and $Q_{k}$ some polynomials with rational coefficients with degree $4(k-2)$. Then, as $Q_{2}$ is a non vanishing constant, the factors of $P_{2}$ define all the center families, that is, $\mathcal{C}_{1}, \mathcal{C}_{2}$, and $\mathcal{C}_{3}^{ \pm}$. In the second case, $P_{k}=a^{2} A^{4 k-5} B_{2}^{2 k-1}\left(A^{2} B_{2}^{2}-C_{1}^{2}\right) Q_{k}\left(B_{2}, C_{1}, C_{2}\right)$, for $k=2,3,4,5$, and $Q_{k}$ some polynomials with rational coefficients with degree $6(k-2)$. Then, also from the factors of $P_{2}$ we have the center families $\mathcal{C}_{1}$ and $\mathcal{F}_{4}^{ \pm}$.

After all the above discussions we can restrict our parameters region to the set $\mathcal{R}=\left\{a \neq 0, B_{2} \neq 0, C_{1} \neq 0, C_{2} \neq 0, C_{2} \neq A B_{2}\right\}$. The proof finishes showing that, computing some crossed resultants as in the previous studies, there are no centers in $\mathcal{R}$. That is, the system of algebraic varieties $\mathcal{S}_{1}=\left\{P_{1}=0, \cdots, P_{5}=0\right\}$, defined in (10), has no solution. From (11) any point in $\mathcal{S}$ also is in $\mathcal{S}_{2}=\left\{R_{2,3}=0, R_{2,4}=\right.$ $\left.0, R_{2,5}=0\right\}$. Straightforward computations show that the two by two resultants of the polynomials in $\mathcal{S}_{2}$ write as

$\operatorname{res}\left(R_{2,3}, R_{2, k}, C_{2}\right)=a^{10 k-8} A^{38 k-42}\left(A^{2}+1\right)^{3 k-2} B_{2}^{40 k-56}(a+A)^{4 k}(a A-1)^{4 k} R_{3, k}(a, A)$, for $k=4,5$, with $R_{3, k}$ polynomials with rational coefficients of degree $70 k-164$. When $a+A=0$ or $a A-1=0$ we compute again the above resultant and we get similar expression but with $R_{3, k}$ polynomials with rational coefficients in $a^{2}$ and degrees 21,34 and 21,32 , respectively. When $a+A \neq 0$ and $a A-1 \neq 0$ we compute the last resultants

$$
\begin{aligned}
\operatorname{res}\left(R_{3,4}, R_{3,5}, a\right)= & A^{2574}\left(16 A^{2}+1\right)\left(A^{2}+16\right)\left(A^{2}+9\right)^{4}\left(9 A^{2}+1\right)^{4}\left(A^{2}+1\right)^{1362} \times \\
& S_{382}\left(A^{2}\right) S_{4843}\left(A^{2}\right), \\
\operatorname{res}\left(R_{3,4}, R_{3,5}, A\right)= & a^{738}\left(25 a^{2}+1\right)^{2}\left(a^{2}+25\right)^{2}\left(a^{2}+9\right)^{16}\left(9 a^{2}+1\right)^{32}\left(a^{2}+1\right)^{1283} \times \\
& \left(1369 a^{4}+1102 a^{2}-171\right)^{2}\left(1038 a^{4}-171 a^{2}-17\right)^{2} \times \\
& \left(321 a^{4}+48 a^{2}+31\right)^{8}\left(T_{6}\left(a^{2}\right)\right)^{2}\left(\hat{T}_{6}\left(a^{2}\right)\right)^{6} T_{13}\left(a^{2}\right)\left(T_{191}\left(a^{2}\right)\right)^{2} T_{4843}\left(a^{2}\right),
\end{aligned}
$$

for some polynomials with rational coefficients $S_{k}, T_{k}$, and $\hat{T}_{k}$ of degree $k$. Consequently, all the possible values for $a$ and $A$ in $\mathcal{S}_{1} \cap \mathcal{R}$ are both algebraic. Using the same argument as in the first part of this proof, about the contradiction with the fact that $\left(A^{*}\right)^{2}=\mathrm{e}^{-\pi a^{*}}=i^{2 i a^{*}}$ is transcendental, the set $\mathcal{S}_{1} \cap \mathcal{R}$ is empty.

Proposition 3.3. System (2) has a center at infinity if the parameters $a^{ \pm}, b^{ \pm}, c^{ \pm}$ belong to the families in Theorem 1.2. 
Proof. All subsystems in families $\mathcal{C}_{1}, \mathcal{C}_{2}$, and $\mathcal{C}_{3}^{ \pm}$are linear centers. Family $\mathcal{C}_{1}$ has a global center because the subsystems defined in regions $\Sigma^{ \pm}$coincide. The singularities of subsystems corresponding to family $\mathcal{C}_{2}$ are visible and located in the straight lines $x-y=0$ and $x+y=0$. Moreover, each one of them is time reversible with respect to the corresponding line. From both symmetries and using the definitions introduced in Section 2 we can check that the first return maps, in each quadrant, are all the identity. So, the composition is also the identity. The system associated to families $\mathcal{C}_{3}^{ \pm}$is time reversible with respect to the straight line $y \pm x=0$.

For the parameters associated to families $\mathcal{F}_{1}$ and $\mathcal{F}_{3}$, the full system (2) is time reversible with respect to $y=0$ and $x=0$, respectively. Then the infinity is a center because in a neighborhood of it the vector field is monodromic. The systems in family $\mathcal{F}_{4}^{-}$can be moved to family $\mathcal{F}_{4}^{+}$changing $\theta$ to $\theta+\pi / 2$ as we have done in Lemma 2.1. Then, only remains the proofs of families $\mathcal{F}_{2}$ and $\mathcal{F}_{4}^{+}$. Both cases are proved similarly in three steps following the ideas of Section 2. First we compute the flying time maps, second we compute the return maps in each quadrant and finally we check that the full composition is the identity. In the family $\mathcal{F}_{2}$, all the flying times are $\pi / 2$ instead of the family $\mathcal{F}_{4}^{+}$that only two of them are. In this last family, we use also that the flying times in $\Sigma^{+}$are $\pi / 2$ and for systems defined in $\Sigma^{-}$the sum is $\pi$.

As we have explained in the introduction we consider only $a^{+}=a>0$.

Family $\mathcal{F}_{2}$ can be expressed, as $A+a \neq 0$, also writing

$$
c^{ \pm}=\mp \frac{A a-1}{A+a} b^{ \pm} .
$$

The return map in the first quadrant can be computed from the parametrized solution of equation (2) in polar coordinates

$$
(x(t), y(t))=\left(-\frac{b^{+}}{A+a}+r \mathrm{e}^{a t} \cos t, \frac{A b^{+}}{A+a}+r \mathrm{e}^{a t} \sin t\right) .
$$

We notice that the equilibrium point belongs to the straight line $A x+y=0$ and, consequently, it is an invisible singularity for system (2) defined in $\Sigma^{+}$.

Let $\tau_{0}, \tau_{0}+\tau_{1}$, be the values of the time such that the solution starts at $(\rho, 0)$ and ends at $\left(0, \rho_{1}\right)$. Substituting the values of $\cos \tau_{0}$ and $\sin \tau_{0}$, from the equations $x\left(\tau_{0}\right)=\rho$ and $y\left(\tau_{0}\right)=0$, in the equation $x\left(\tau_{0}+\tau_{1}\right)=0$ we obtain that $\tau_{1}$ satisfies $f_{\rho}\left(\tau_{1}\right)=0$ with

$$
f_{\rho}(\tau)=\rho \mathrm{e}^{a \tau} \cos \tau+\frac{b^{+}}{A+a}\left(A \mathrm{e}^{a \tau} \sin \tau+\mathrm{e}^{a \tau} \cos \tau-1\right) .
$$

We observe that $f_{\rho}(\pi / 2)=0$ and, if this value is the smallest positive zero of $f_{\rho}$, we can obtain

$$
\rho_{1}=y\left(\tau_{0}+\pi / 2\right)=\frac{\rho}{A}+\frac{b^{+}\left(A^{2}+1\right)}{A(A+a)} .
$$

The solution in $\Sigma^{+}$defining $\Pi_{1}$ can be seen in Figure 2 (right).

The property $f_{\rho}(\tau) \neq 0$ for $\tau \in[0, \pi / 2)$ follows from

$$
f_{\rho}(0)=\rho, \quad f_{\rho}^{\prime}(0)=a \rho+b^{+}, \quad f_{\rho}^{\prime}(\pi / 2)=-\frac{\rho}{A}+\frac{(A a-1) b^{+}}{A(A+a)},
$$



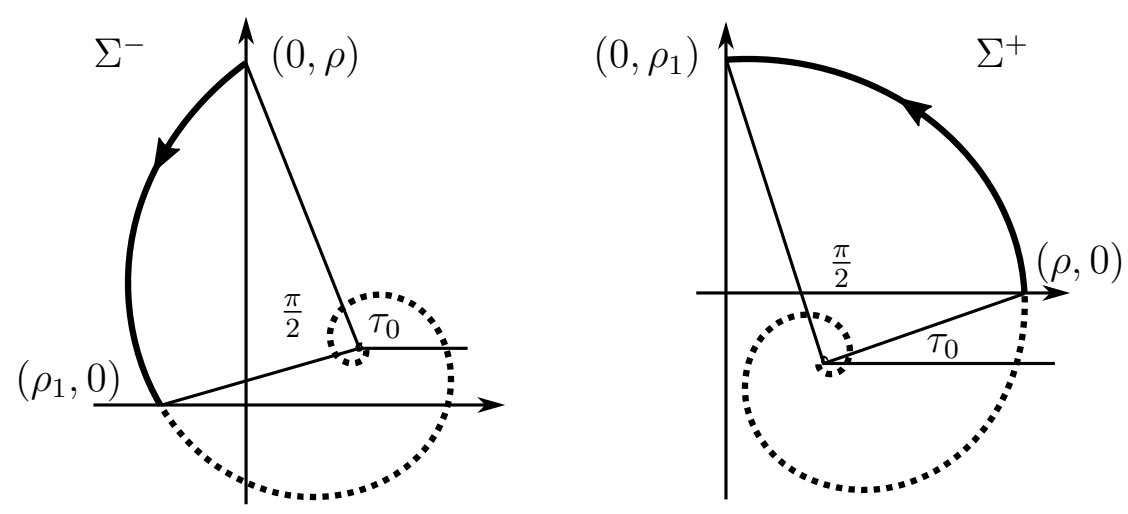

FiguRE 2. Representation of the return maps $\Pi_{1}$ (right) and $\Pi_{2}$ (left).

and the fact that the derivative

$$
f_{\rho}^{\prime}(\tau)=\mathrm{e}^{a \tau}(a \cos \tau-\sin \tau) \rho+b^{+} \frac{\mathrm{e}^{a \tau}}{A+a}((A a-1) \sin \tau+(A+a) \cos \tau)
$$

vanishes only at $\tau=0$ for $a=0$ and has exactly one positive zero for $a>0$. See Figure 3 (left).
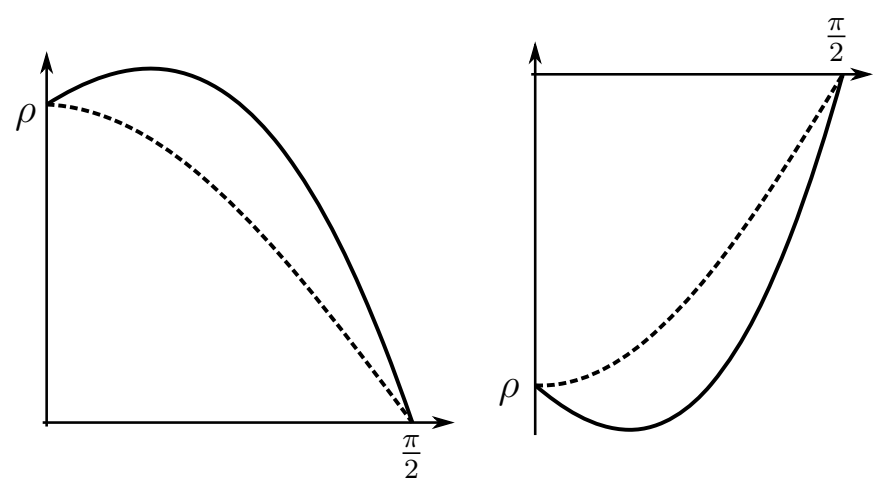

FiguRE 3. The graphs of $f_{\rho}$ for $\rho>0$ (left) and $\rho<0$ (right), for different values of $a>0, a=0$ (dash).

As $\rho_{1}>0$, from the definition in Section 2 and (15), we get that the return map in the first quadrant is the affine map

$$
\Pi_{1}(\rho)=\frac{\rho}{A}+\frac{b^{+}\left(A^{2}+1\right)}{A(A+a)} .
$$

The study for the return map in the third quadrant coincides with above discussion, taking the solution passing through $(\rho, 0)$ and $\left(0, \rho_{1}\right)$, with $\rho<0$ and $\rho_{1}<0$. Because the above procedure works also for negative values of $\rho$. See the plot of the function $f_{\rho}$ for this case in Figure 3 (right). The definition of $\Pi_{3}$ in Section 2 needs the positiveness of $\rho$ and $\rho_{1}$. Changing adequately the signs in (15) we get

$$
\Pi_{3}(\rho)=\frac{\rho}{A}-\frac{b^{+}\left(A^{2}+1\right)}{A(A+a)} .
$$

The return maps in the second and fourth quadrant can be obtained similarly taking the solution of equation (2) passing through $(0, \rho)$ and $\left(\rho_{1}, 0\right)$. The equilibrium 
point, that belongs to the straight line $-A x+y$, is also invisible for system (2) defined in $\Sigma^{-}$. Equations (13), (14), and (16) change to

$$
\begin{aligned}
& (x(t), y(t))=\left(\frac{b^{-}}{A+a}+r \mathrm{e}^{-a t} \cos t, \frac{A b^{-}}{A+a}+r \mathrm{e}^{-a t} \sin t\right), \\
& g_{\rho}(\tau)=\rho \mathrm{e}^{-a \tau} \cos \tau-\frac{b^{-}}{A+a}\left(A \mathrm{e}^{-a \tau} \cos \tau+\mathrm{e}^{-a \tau} \sin \tau-A\right)
\end{aligned}
$$

and

$$
\rho_{1}=y\left(\tau_{0}+\pi / 2\right)=-A \rho+\frac{b^{-}\left(A^{2}+1\right)}{A+a} .
$$

The solution in $\Sigma^{-}$defining $\Pi_{2}$ can be seen in Figure 2 (left). Using the definitions of the return maps in Section 2 but with distances, changing adequately the signs in (18), we get

$$
\Pi_{2}(\rho)=A \rho-\frac{b^{-}\left(A^{2}+1\right)}{A+a}, \quad \Pi_{4}(\rho)=A \rho+\frac{b^{-}\left(A^{2}+1\right)}{A+a} .
$$

The proof of this family finishes straightforward checking, using (16), (17), and (19), that the composition $\Pi_{4} \circ \Pi_{3} \circ \Pi_{2} \circ \Pi_{1}$ is the identity map.

The study of the centers in family $\mathcal{F}_{4}^{+}$starts obtaining the equivalent relations

$$
b^{-}=\frac{A-a}{A+a} b^{+}, \quad c^{ \pm}=\mp \frac{A a \mp 1}{A+a} b^{+} .
$$

We notice that the definition of $b^{+}$coincides with the one in (12). Then, the return maps $\Pi_{1}$ and $\Pi_{3}$, are the same as the previous family, defined in (16) and (17). Consequently, only the computation of $\Pi_{2}$ and $\Pi_{4}$ are necessary to be done. In a similar way, the solution of (2) in the second quadrant writes as

$$
(x(t), y(t))=\left(-\frac{b^{+}}{A+a}+r \mathrm{e}^{-a t} \cos t, \frac{A b^{+}}{A+a}+r \mathrm{e}^{-a t} \sin t\right) .
$$

The main difference with respect to family $\mathcal{F}_{2}$ is in the flying times from a point in $x=0$ to $y=0$, that they are not $\pi / 2$. For this family the equilibrium point of system (2) defined in $\Sigma^{-}$is visible and it coincides with the one, which is invisible, defined in $\Sigma^{+}$also for system (2).

Let us consider the solution of (2), defined in the second quadrant, starting at $\left(0, \pi_{1}\right)$ and finishing at $\left(\pi_{2}, 0\right)$, being $\tau_{1}$ the corresponding flying time. See Figure 4. Straightforward computations get

$$
\mathrm{e}^{-a \tau_{1}}(A+a) \rho \cos \tau_{1}+b^{+}\left(A \mathrm{e}^{-a \tau_{1}} \sin \tau_{1}+\mathrm{e}^{-a \tau_{1}} \cos \tau_{1}+A^{2}\right)=0
$$

and

$$
\pi_{2}=-\frac{1}{A} \mathrm{e}^{-a \tau_{1}} \rho \sin \tau_{1}-\frac{b^{+}}{A(A+a)}\left(\mathrm{e}^{-a \tau_{1}} \sin \tau_{1}-A \mathrm{e}^{-a \tau_{1}} \cos \tau_{1}+A\right) .
$$

Using the function $\Pi_{3}$, defined in $(17)$, we can compute the image, $\left(0, \pi_{3}\right)$, of the point $\left(\pi_{2}, 0\right)$ with the third return map,

$$
\pi_{3}=-\frac{1}{A^{2}} \mathrm{e}^{-a \tau_{1}} \rho \sin \tau_{1}-\frac{b^{+}}{A^{2}(A+a)}\left(\mathrm{e}^{-a \tau_{1}} \sin \tau_{1}-A \mathrm{e}^{-a \tau_{1}} \cos \tau_{1}-A^{3}\right) .
$$



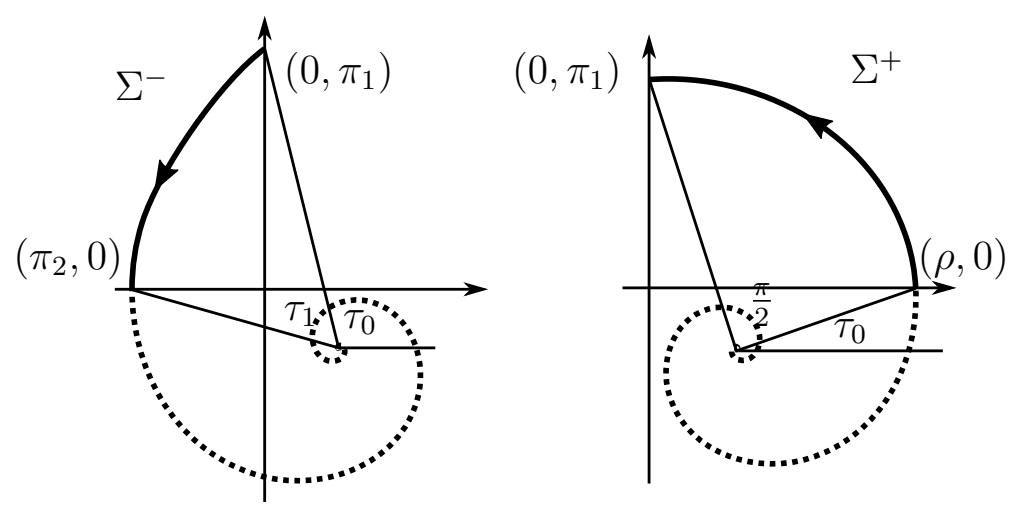

Figure 4. Representation of the return maps $\Pi_{1}$ (right) and $\Pi_{2}$ (left).

For the last step, we consider also the solution $(20)$ from the point $\left(0, \pi_{3}\right)$ to $\left(\pi_{4}, 0\right)$ with flying time $\tau_{2}$. The simplified equivalent equation (14) for the flying $\tau_{2}$, using that $2 \pi$ is the total flying time and so, $\tau_{1}+\tau_{2}=\pi$, is

$$
(A+a) \sin \left(\tau_{1}-\tau_{2}\right) \rho+b^{+}\left(\sin \left(\tau_{1}-\tau_{2}\right)-A \cos \left(\tau_{1}-\tau_{2}\right)-2 \mathrm{e}^{-a \tau_{2}} \sin \tau_{2}-A\right)=0,
$$

and

$\pi_{4}=\frac{1}{2}\left(\cos \left(\tau_{1}-\tau_{2}\right)+1\right) \rho+\frac{b^{+}}{2(A+a)}\left(A \sin \left(\tau_{1}-\tau_{2}\right)+\cos \left(\tau_{1}-\tau_{2}\right)+2 \mathrm{e}^{-a \tau_{2}} \cos \tau_{2}-1\right)$.

Finally, we check, using the equations (21) and (22) and also that $\tau_{1}+\tau_{2}=\pi$, that $\pi_{4}=\rho$. As the composition of the four return maps is the identity, family $\mathcal{F}_{4}^{+}$defines a center at infinity as we wanted to prove.

\section{Five Limit CYCLES IN A 4-STAR-SYMMETRIC SYSTEM}

This section is devoted to the proof of Theorem 1.1. From Proposition 2.3 we can restrict our analysis to $a^{+}+a^{-}=0$. Under this condition, Proposition 4.2 will show that system (2) exhibits a weak focus of order 5 that unfolds 4 limit cycles. When this condition is broken an extra limit cycles appear and Theorem 1.1 follows. As in the previous section we denote $a^{+}=-a^{-}=a$. We notice that with the change of variables (3), the infinity is moved to the origin. With the same procedure, we could obtain other results showing that there exist systems with weak foci of order $k$ bifurcating $k$ limit cycles for $k=2,3,4$.

From the proof of Proposition 3.2 it follows the next corollary.

Corollary 4.1. There are no parameter values $\left(a^{ \pm}, b^{ \pm}, c^{ \pm}\right)$such that system (4) has a weak focus of order higher than five at the origin.

Proof. The proof follows checking all the cases appearing in the proof of Proposition 3.2. More concretely, under the change of parameters (7), when $B_{2}=0$ and $C_{2} \neq 0$ the order of the weak focus is one. If $B_{2}=C_{1}=0$ then the maximum weak focus order is four. Only when $B_{2} \neq 0$ weak foci of order 5 appear. But when the first five Lyapunov quantities obtained in Lemma 3.1 vanish we get centers of (2).

Next result exhibits the maximum number of limit cycles bifurcating from the origin for system (4) under the condition $a^{+}+a^{-}=0$. 
Proposition 4.2. Let us consider system (4) under the conditions $a^{+}=-a^{-}=a$. Then, there exist values of the parameters $a, b^{ \pm}$, and $c^{ \pm}$such that system (4) has a weak focus at the origin of order 5 that unfolds 4 limit cycles.

The proof follows fixing an specific value for one of the five free parameters and proving that the varieties $\left\{L_{1}=L_{2}=L_{3}=L_{4}=0\right\}$ intersects in, at least, one point in $\mathbb{R}^{4}$. This is proved using the Poincaré-Miranda Theorem that we recall here by completeness. This technical result is a generalization of Bolzano's Theorem for higher dimensions. Moreover, it is also necessary to prove that, at this point, the intersection is transversal and $L_{5}$ is different from zero.

Theorem $4.3([20])$. Let $f: \mathbb{R}^{n} \rightarrow \mathbb{R}^{n}, f=\left(f_{1}, \ldots, f_{n}\right)$, be a continuous map such that for each $1 \leq i \leq n, f_{i}\left(I_{i}^{-}\right) \subset(-\infty, 0]$ and $f_{i}\left(I_{i}^{+}\right) \subset[0, \infty)$. Then there exists a point $\zeta \in I^{n}$ such that $f(\zeta)=0$. Where $I^{n}=[-h, h]^{n}$, and $I_{i}^{ \pm}=\left\{\left(x_{1}, \ldots, x_{n}\right) \in\right.$ $\left.I^{n}: x_{i}= \pm h\right\}$, for some positive value $h$.

Next we will prove the main result of this section.

Proof of Proposition 4.2. We start doing the change in the parameters space (7) as in Proposition 3.2 and fixing the value $B_{2}=10$. Then, we have only four parameters $\left(a, B_{1}, C_{1}, C_{2}\right)$ and we define the function $f=\left(L_{1}, L_{2}, L_{3}, L_{4}\right)$. The proof is given in two steps. First, we provide a box $I^{4}$ in Theorem 4.3 such that, it exists a point, $\zeta^{*}=\left(a^{*}, B_{1}^{*}, C_{1}^{*}, C_{2}^{*}\right)$, satisfying $f\left(\zeta^{*}\right)=0$. Second, we check that the next Lyapunov quantity and the determinant of the Jacobian matrix of $f$, at every point in the box $I^{4}$, are different from zero. In particular, $L_{5}\left(\zeta^{*}\right) \neq 0$ and $D f_{\left(a, B_{1}, C_{1}, C_{2}\right)}\left(\zeta^{*}\right) \neq 0$. We will check such conditions first numerically and then analytically. More concretely, we do a computer assisted proof to prove the existence of a transversal intersection point by means of an accurate rational interval analysis.

From Lemma 3.1 we compute the first Lyapunov quantities $L_{k}, k=1, \ldots, 5$. Then, as $a^{+}+a^{-}=0$, we have $L_{0}=0$. As in the previous results, we denote $a^{+}=-a^{-}=a$ and $A=\mathrm{e}^{-\frac{\pi}{2} a}$.

Solving $L_{1}=L_{2}=0$ in (9), with the condition $C_{1}\left(10 A-C_{2}\right) \neq 0$, we get

$$
B_{1}=\frac{C_{1} C_{2}}{10 A^{2}}, \quad C_{1}^{2}=\frac{A Q_{1}}{Q_{2}},
$$

where $Q_{1}=Q_{1}\left(a, A, C_{2}\right)$ and $Q_{2}=Q_{2}\left(a, A, C_{2}\right)$ are the polynomials

$$
\begin{aligned}
Q_{1}= & 10^{2}(3 A+4 a) C_{2}^{3}-10^{3} A\left(9 A^{2} a+8 A a^{2}-3 A-a\right) C_{2}^{2} \\
& +10^{4} A^{2} a\left(3 A^{2}+A a+1\right) C_{2}+10^{5} A^{4} a^{2}, \\
Q_{2}= & (-4 A a+3) C_{2}^{3}-10\left(A^{2} a+8 A a^{2}-3 A-9 a\right) C_{2}^{2} \\
& -10^{2} A a\left(A^{2}-A a+3\right) C_{2}+10^{3} A^{3} a^{2} .
\end{aligned}
$$

Clearly necessary conditions are that $Q_{2}$ in (23) should be different from zero and the quotient $Q_{1} / Q_{2}$ be positive.

Using the above relations we get

$$
\begin{aligned}
& L_{3}=C_{1} C_{2} a A^{-4}\left(A^{2}+1\right)\left(10 A-C_{2}\right)^{3} Q_{2}^{-2} P_{3}\left(a, A, C_{2}\right), \\
& L_{4}=C_{1} C_{2} a A^{-7}\left(A^{2}+1\right)\left(10 A-C_{2}\right)^{3} Q_{2}^{-3} P_{4}\left(a, A, C_{2}\right), \\
& L_{5}=C_{1} C_{2} a A^{-10}\left(A^{2}+1\right)\left(10 A-C_{2}\right)^{3} Q_{2}^{-4} P_{5}\left(a, A, C_{2}\right),
\end{aligned}
$$


where $P_{k}$ are polynomials with rational coefficients of degrees $16,28,40$ for $k=$ $3,4,5$, respectively. We do not write here the expressions of such polynomials because of their size. In fact, they have 104, 489, and 1313 monomials, respectively. Consequently, we can use $\left(L_{1}, L_{2}, P_{3}, P_{4}, P_{5}\right)$ instead of $\left(L_{1}, L_{2}, L_{3}, L_{4}, L_{5}\right)$ and the equivalent system of equations $\widetilde{\mathcal{S}}=\left\{L_{1}=L_{2}=P_{3}=P_{4}=0\right\}$. The other factors in (24) are not considered because they vanish identically all the equations.

As $A=\mathrm{e}^{-a \pi / 2}$, then a weak focus point of order five is given, from (24), by a solution $\left(a^{*}, C_{2}^{*}\right)$ of the system of two equations

$$
\left\{P_{3}=P_{4}=0\right\}
$$

with only two unknowns $\left(a, C_{2}\right)$ such that $P_{5}\left(a^{*}, A^{*}, C_{2}^{*}\right) \neq 0$ and the necessary condition $Q_{1}\left(a^{*}, A^{*}, C_{2}^{*}\right) Q_{2}\left(a^{*}, A^{*}, C_{2}^{*}\right)>0$, where $A^{*}=\mathrm{e}^{-a^{*} \pi / 2}$. From (23) we get the corresponding values for $C_{1}^{*}$ and $B_{1}^{*}$. Straightforward computations show that the Jacobian matrix of the function $\mathcal{P}:\left(a, B_{1}, C_{1}, C_{2}\right) \mapsto\left(L_{1}, L_{2}, P_{3}, P_{4}\right)$ is

$$
D \mathcal{P}\left(a^{*}, B_{1}^{*}, C_{1}^{*}, C_{2}^{*}\right)=2 A^{2} C_{1}^{*} Q_{2}\left(a^{*}, A^{*}, C_{2}^{*}\right) D\left(P_{3}, P_{4}\right)_{\left(a, C_{2}\right)}\left(a^{*}, A^{*}, C_{2}^{*}\right) .
$$

Then, the transversality of the varieties defined in system $\widetilde{\mathcal{S}}$ is a direct consequence of the nonvanishing Jacobian

$$
J=D\left(P_{3}, P_{4}\right)_{\left(a, C_{2}\right)}\left(a^{*}, A^{*}, C_{2}^{*}\right) \neq 0 .
$$

We point out that the above function is a polynomial in $\left(a, A, C_{2}\right)$ of degree 43 with 3180 monomials.

In Figure 5 we have drawn the zero level curves $\left\{P_{3}=0\right\}$ and $\left\{P_{4}=0\right\}$ in different zones of the reduced parameter space $\left(a, C_{2}\right)$. It is shown also some zooms to see better, at least, one transversal intersection satisfying the necessary conditions for the existence of a transversal intersection point in the complete parameter space $\left(a, B_{2}, C_{1}, C_{2}\right)$, together with the condition $Q_{1} Q_{2}>0$,
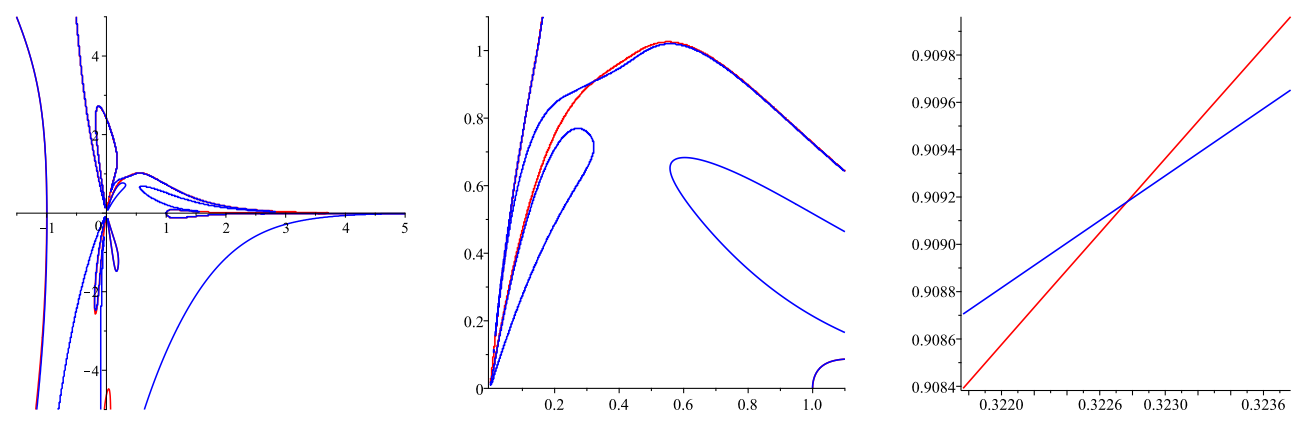

Figure 5. The drawing of the zero level curves $\left\{P_{3}=0\right\}$ and $\left\{P_{4}=\right.$ $0\}$ in red and blue, respectively, in the parameter space $\left(a, C_{2}\right)$

Numerically, working with enough accuracy, we can get

$$
\left(a^{*}, A^{*}, B_{1}^{*}, C_{1}^{*}, C_{2}^{*}\right) \approx(0.322766,0.602300,8.496686,33.902075,0.909179) .
$$

Additionally,

$$
\begin{array}{ll}
Q_{1}\left(a^{*}, C_{2}^{*}\right)=4.31954 \cdot 10^{3}, & Q_{2}\left(a^{*}, C_{2}^{*}\right)=2.26359, \\
P_{5}\left(a^{*}, C_{2}^{*}\right)=-2.03342 \cdot 10^{10}, \quad J\left(a^{*}, C_{2}^{*}\right)=3.30868 \cdot 10^{12} .
\end{array}
$$

We remark that the chosen point also satisfies the necessary conditions such that $B_{1}$ and $C_{1}$, defined in (23), exist. This is not true for all intersection points. 
We can recover the original parameters, with the inverse change of (7),

$$
b^{ \pm}=\frac{a\left(B_{1} \mp B_{2}\right)+C_{1} \mp C_{2}}{2\left(a^{2}+1\right)}, \quad c^{ \pm}=\frac{\mp a\left(C_{1}+C_{2}\right) \pm B_{1}-B_{2}}{2\left(a^{2}+1\right)} .
$$

Then, the origin of system (4) for $\left(a^{*}, b_{*}^{+}, c_{*}^{+}, b_{*}^{-}, c_{*}^{-}\right)$is a weak focus of order 5 . Substituting (26) in the above expression we get

$$
\left(a^{*}, b_{*}^{+}, c_{*}^{+}, b_{*}^{-}, c_{*}^{-}\right) \approx(0.3227657,14.72030,-5.502868,18.46683,-3.287880) .
$$

So, at this point, numerically the first four Lyapunov quantities vanish transversally and $L_{5}\left(a^{*}, b_{*}^{+}, c_{*}^{+}, b_{*}^{-}, c_{*}^{-}\right) \approx-2.0197688 \cdot 10^{8}$.

Next, we prove analytically the existence of the point (26), equivalently (28), satisfying all the conditions described above.

We start writing $\left(\mathrm{e}^{a \pi / 2}\right)^{-1}$ instead $\mathrm{e}^{-a \pi / 2}$ in all the expressions involved in the computations because they are simpler when the exponential function is increasing. In order to apply Theorem 4.3 to system (25) we perform the change of variables

$$
\begin{aligned}
a & =\frac{363394237984}{1125876185023}-\frac{14774107617}{61873882348312} u-\frac{53184397}{13103427838970537} v, \\
C_{2} & =\frac{2250212532359}{2474994238126}-\frac{2109559243}{18668085007969} u-\frac{20674157 v}{6487787928613874} v .
\end{aligned}
$$

Then, we get $P_{3}(u, v) \approx u+O_{2}(u, v)$ and $P_{4}(u, v) \approx v+O_{2}(u, v)$. With this change of variables, the involved functions, $P_{3}, P_{4}, P_{5}, Q_{1}, Q_{2}$, and $J$, are all polynomials with rational coefficients in the variables $\pi, u, v$, and $\mathrm{e}^{\pi\left(x_{0}+x_{1} u+x_{2} v\right)}$, with $x_{0}=-181697118992 / 1125876185023, x_{1}=14774107617 / 123747764696624$, and $x_{2}=53184397 / 26206855677941074$.

The existence of an intersection point, $\left(u^{*}, v^{*}\right)$, follows finding an appropriate value for $h$ satisfying the hypotheses of Theorem 4.3. For $h=10^{-7}$, it can be checked numerically that

$$
\begin{aligned}
& \max P_{3}(-h, v)<0, \min P_{3}(h, v)>0 \text { for } v \in[-h, h], \\
& \max P_{4}(u,-h)<0, \min P_{4}(u, h)>0 \text { for } u \in[-h, h] .
\end{aligned}
$$

Additionally,

$$
\min Q_{1}(u, v)>0, \min Q_{2}(u, v)>0, \max P_{5}(u, v)<0, \min J(u, v)>0,
$$

for $(u, v) \in[-h, h]^{2}$. We can recover the existence of the point (26) from (29) and (23). The transversality and the existence of a weak focus of order 5, at least numerically, is also clear.

The analytical proof of the above properties can be done using arithmetic interval analysis with $h=10^{-9}$. We will use the technical results of the last section with the polynomial approximation for the exponential function given by $m=21$, see Proposition 5.1. For $\pi$, we take

$$
\underline{\pi}=\frac{21053343141}{6701487259} \leq \pi \leq \frac{1783366216531}{567663097408}=\bar{\pi}
$$

from its continuous fraction expression with 22 and 23 partial quotients.

First we prove (30) and second (31). With the change (29) we write

$$
\begin{array}{ll}
P_{3}(-h, v)=g_{1}\left(v, \mathrm{e}^{\pi\left(y_{0}^{-}+y_{1}^{-} v\right)}\right)=: \hat{g}_{1}(v), & P_{3}(h, v)=g_{2}\left(v, \mathrm{e}^{\pi\left(y_{0}^{+}+y_{1}^{+} v\right)}\right)=: \hat{g}_{2}(v), \\
P_{4}(u,-h)=g_{3}\left(u, \mathrm{e}^{\pi\left(z_{0}^{-}+z_{1}^{-} u\right)}\right)=: \hat{g}_{3}(u), \quad P_{4}(u, h)=g_{4}\left(u, \mathrm{e}^{\pi\left(z_{0}^{+}+z_{1}^{+} u\right)}\right)=: \hat{g}_{4}(u),
\end{array}
$$


with $y_{0}^{-}=x_{0}-x_{1} h, y_{1}^{-}=x_{2}, y_{0}^{+}=x_{0}+x_{1} h, y_{1}^{+}=x_{2}, z_{0}^{-}=x_{0}-x_{2} h, z_{1}^{-}=x_{1}$, $z_{0}^{+}=x_{0}+x_{2} h$, and $z_{0}^{+}=x_{1}$ all rational numbers. We do not detail the explicit expressions of the functions $g_{1}(x, y), g_{2}(x, y), g_{3}(x, y)$, and $g_{4}(x, y)$ because of their size. They are polynomials with rational coefficients of degrees 16, 16, 28, and 28 with 118, 118, 332, and 332 monomials in $(x, y)$, respectively. Then applying Proposition 5.2 we have that

$$
\begin{aligned}
& \hat{g}_{1}([-h, h]) \subset\left[\eta_{1}, \xi_{1}\right] \approx\left[-1.0084558 \cdot 10^{-9},-9.9154416 \cdot 10^{-10}\right], \\
& \hat{g}_{2}([-h, h]) \subset\left[\eta_{2}, \xi_{2}\right] \approx\left[9.9154416 \cdot 10^{-10}, 1.0084558 \cdot 10^{-9}\right] \text {, } \\
& \hat{g}_{3}(-h) \in\left[\eta_{3}, \xi_{3}\right] \approx\left[-1.09345834416 \cdot 10^{-9},-9.06375115265 \cdot 10^{-10}\right], \\
& \hat{g}_{3}(-h)+2 h \hat{g}_{3}^{\prime}(-h) \in\left[\eta_{4}, \xi_{4}\right] \approx\left[-1.09345830474 \cdot 10^{-9},-9.06375075847 \cdot 10^{-10}\right] \text {, } \\
& \hat{g}_{3}^{\prime \prime}([-h, h]) \subset\left[\eta_{5}, \xi_{5}\right] \approx[-19.7094899356371570,-19.7091168953367466], \\
& \hat{g}_{4}(-h) \in\left[\eta_{6}, \xi_{6}\right] \approx\left[9.065416558399 \cdot 10^{-10}, 1.093624884733879 \cdot 10^{-9}\right], \\
& \hat{g}_{4}(h) \in\left[\eta_{7}, \xi_{7}\right] \approx\left[9.065416558414 \cdot 10^{-10}, 1.093624884735517 \cdot 10^{-9}\right], \\
& \hat{g}_{4}^{\prime \prime}([-h, h]) \subset\left[\eta_{8}, \xi_{8}\right] \approx[-19.7094899356371588,-19.7091168953367484] \text {. }
\end{aligned}
$$

The above expressions prove (30). We remark that for $\hat{g}_{3}$ and $\hat{g}_{4}$ we have used the extreme values of the interval of definition, the sign of the second derivative and the value of the straight line tangent at $x=-h$ evaluated at $x=h$. This is because the lower and upper bounds obtained with the results in the last section for $\hat{g}_{3}([-h, h])$ and $\hat{g}_{4}([-h, h])$ do not prove directly $(30)$.

Next we prove (31). As above, with the change (29) we write

$$
\begin{array}{ll}
Q_{1}(u, v)=g_{5}\left(u, v, \mathrm{e}^{\pi\left(x_{0}+x_{1} u+x_{2} v\right)}\right), & Q_{2}(u, v)=g_{6}\left(u, v, \mathrm{e}^{\pi\left(x_{0}+x_{1} u+x_{2} v\right)}\right), \\
P_{5}(u, v)=g_{7}\left(u, v, \mathrm{e}^{\pi\left(x_{0}+x_{1} u+x_{2} v\right)}\right), & J(u, v)=g_{8}\left(u, v, \mathrm{e}^{\pi\left(x_{0}+x_{1} u+x_{2} v\right)}\right),
\end{array}
$$

where $g_{5}(x, y, z), g_{6}(x, y, z)$, and $g_{7}(x, y, z)$ are polynomials with rational coefficients of degrees 6, 5, and 40 and 56, 41, 8494 monomials, respectively. The last $g_{8}(x, y, z)$ is also a polynomial, of degree 43 and 20695 monomials, but the coefficients are polynomials of degree 1 in $\pi$ also with rational coefficients. Applying Proposition 5.2 we have

$$
\begin{aligned}
Q_{1}\left([-h, h]^{2}\right) & \subset\left[\eta_{9}, \xi_{9}\right] \approx\left[4.3195435234081 \cdot 10^{3}, 4.3195435234277 \cdot 10^{3}\right], \\
Q_{2}\left([-h, h]^{2}\right) & \subset\left[\eta_{10}, \xi_{10}\right] \approx[2.263592961126,2.263592961413], \\
P_{5}\left([-h, h]^{2}\right) & \subset\left[\eta_{11}, \xi_{11}\right] \approx\left[-2.03344613 \cdot 10^{10},-2.03340080 \cdot 10^{10}\right], \\
J\left([-h, h]^{2}\right) & \subset\left[\eta_{12}, \xi_{12}\right] \approx\left[3.30867903 \cdot 10^{12}, 3.30868520 \cdot 10^{12}\right] .
\end{aligned}
$$

We notice that the numerical values (27) agree with the above approximations.

Finally, we remark that with higher values for $h$ we have not been able to prove the properties (30) and (31). Moreover, with smaller values for $h$ the lower and upper bounds for the images of $\hat{g}_{3}([-h, h])$ and $\hat{g}_{4}([-h, h])$ obtained with Proposition 5.2 do not prove the sign properties (30) for $P_{4}$. This is why we have used all properties $(32)$

\section{Appendix. Bounding a exponential-Polynomial functions}

Let us denote $u=\left(u_{1}, \ldots, u_{n}\right), b=\left(b_{1}, \ldots, b_{n}\right)$, and $\ell=\left(\ell_{0}, \ell_{1}, \ldots, \ell_{n}, \ell_{n+1}\right)$. In this section we provide rational lower and upper bounds for polynomials in the 
variables $u, \pi$, and $\mathrm{e}^{\pi(a+b \cdot u)}$ with rational coefficients when $a<0$ and $b_{i} \geq 0$. That is, each monomial can be written as

$$
A_{\ell} M_{\ell}(u)=A_{\ell} \mathrm{e}^{\pi \ell_{0}(a+b \cdot u)} u_{1}^{\ell_{1}} \cdots u_{n}^{\ell_{n}} \pi^{\ell_{n+1}},
$$

where $b \cdot u=\sum_{i=1}^{n} b_{i} u_{i}$. We notice that $u \in[-h, h]^{n}$ for a rational number $h$. Moreover, the value $a$, the coefficients $A_{\ell}$ and the components of $b$ are all rational numbers and the components of $\ell$ are nonnegative integer numbers.

The following straightforward results provide rational lower and upper bounds for the principal functions in the above monomial and for the complete polynomial when $n=0,1$, and 2 . They can be easily implemented in any computer algebra system.

Proposition 5.1. Let $m$ be a natural number, $h>0$ be a rational number, and $\underline{\pi}, \bar{\pi}$ be two rational numbers such that $\underline{\pi}<\pi<\bar{\pi}$. Then the next properties hold:

(i) For $-1 \leq a \pi \leq 0$, we have

$$
\underline{E}(a):=\left(E_{m}(-\bar{\pi} a, 3)\right)^{-1} \leq \mathrm{e}^{\pi a} \leq\left(E_{m}(-\underline{\pi} a, 0)\right)^{-1}=: \bar{E}(a),
$$

where $E_{m}(x, \tau)=\sum_{j=0}^{m} \frac{x^{j}}{j !}+\tau \frac{x^{m+1}}{(m+1) !}$.

(ii) When $M_{\ell}=\mathrm{e}^{\pi \ell_{0} a} \pi^{\ell_{1}}$ and $-1 \leq a \pi \leq 0$, then

$$
\underline{M_{\ell}}:=(\underline{E}(a))^{\ell_{0}}{\underline{\ell^{\ell_{1}}}} \leq M_{\ell} \leq(\bar{E}(a))^{\ell_{0}} \bar{\pi}^{\ell_{1}}=: \overline{M_{\ell}} .
$$

(iii) When $M_{\ell}(u)=\mathrm{e}^{\pi \ell_{0}\left(a+b_{1} u_{1}\right)} u_{1}^{\ell_{1}} \pi^{\ell_{2}},-1 \leq\left(a+b_{1} h\right) \pi \leq 0,-1 \leq\left(a-b_{1} h\right) \pi \leq 0$, and $\ell_{1}-h \ell_{0} b_{1}>0$ then

$$
\underline{M_{\ell}(u)} \leq M_{\ell}(u) \leq\left(\bar{E}\left(a+b_{1} h\right)\right)^{\ell_{0}} h^{\ell_{1}} \bar{\pi}^{\ell_{2}}=: \overline{M_{\ell}(u)},
$$

where

$$
\underline{M_{\ell}(u)}= \begin{cases}\left(\underline{E}\left(a-b_{1} h\right)\right)^{\ell_{0}} \pi^{\ell_{2}} & \ell_{1}=0, \\ -\left(\bar{E}\left(a-b_{1} h\right)\right)^{\ell_{0}} h^{\ell_{1}} \bar{\pi}^{\ell_{2}} & \ell_{1} \text { odd, } \\ 0 & \ell_{1}>0, \text { even } .\end{cases}
$$

(iv) When $M_{\ell}(u)=\mathrm{e}^{\pi \ell_{0}\left(a+b_{1} u_{1}+b_{2} u_{2}\right)} u_{1}^{\ell_{1}} u_{2}^{\ell_{2}} \pi^{\ell_{3}},-1 \leq\left(a+b_{1} h+b_{2} h\right) \pi \leq 0,-1 \leq$ $\left(a-b_{1} h-b_{2} h\right) \pi \leq 0, \ell_{1}-h \ell_{0} b_{1}>0$ and $\ell_{2}-h \ell_{0} b_{2}>0$ then

$$
\underline{M_{\ell}(u)} \leq M_{\ell}(u) \leq\left(\bar{E}\left(a+b_{1} h+b_{2} h\right)\right)^{\ell_{0}} h^{\ell_{1}+\ell_{2}} \bar{\pi}^{\ell_{3}}=: \overline{M_{\ell}(u)},
$$

where

$$
\underline{M_{\ell}(u)}=\left\{\begin{array}{lc}
\left(\underline{E}\left(a-b_{1} h-b_{2} h\right)\right)^{\ell_{0}} \underline{\pi}^{\ell_{3}} & \ell_{1}=\ell_{2}=0, \\
-\left(\bar{E}\left(a-\left(b_{1}-b_{2}\right) h\right)\right)^{\ell_{0}} h^{\ell_{1}+\ell_{2}} \bar{\pi}^{\ell_{3}} & \left(\ell_{1}, \ell_{2} \text { both odd and } b_{2} \geq b_{1}\right) \\
& \text { or }\left(\ell_{1} \text { odd }, \ell_{2} \text { even }\right), \\
-\left(\bar{E}\left(a-\left(b_{2}-b_{1}\right) h\right)\right)^{\ell_{0}} h^{\ell_{1}+\ell_{2}} \bar{\pi}^{\ell_{3}} & \left(\ell_{1}, \ell_{2} \text { both odd and } b_{1} \geq b_{2}\right) \\
0 & \text { or }\left(\ell_{1} \text { even }, \ell_{2} \text { odd }\right), \\
0 & \ell_{1}>0, \ell_{2}>0, \text { both even } .
\end{array}\right.
$$

Proposition 5.2. Let $A_{\ell}$ be rational numbers and $M_{\ell}$ and $M_{\ell}(u)$ be monomials, satisfying the hypotheses of Proposition 5.1. If $a<0$ and $b_{i} \geq 0$, for $i=1, \ldots, n$, then there exists small enough rational number $h>0$ such that the next properties hold. 
(i) If $F=\sum_{\ell=0}^{d} A_{\ell} M_{\ell}$ where $M_{\ell}=\mathrm{e}^{\pi \ell_{0} a} \pi^{\ell_{1}}$

$$
F \in[\eta, \xi]:=\left[\sum_{\ell=0}^{d} A_{\ell} \mathcal{L}\left(A_{\ell}, M_{\ell}\right), \sum_{\ell=0}^{d} A_{\ell} \mathcal{U}\left(A_{\ell}, M_{\ell}\right)\right] .
$$

(ii) If $F(u)=\sum_{\ell=0}^{d} A_{\ell} M_{\ell}(u)$ where $M_{\ell}(u)=\mathrm{e}^{\pi \ell_{0}(a+b \cdot u)} u_{1}^{\ell_{1}} \cdots u_{n}^{\ell_{n}} \pi^{\ell_{n+1}}$ and $n=1$ or $n=2$, then

$$
F\left([-h, h]^{n}\right) \subset[\eta, \xi]:=\left[\sum_{\ell=0}^{d} A_{\ell} \mathcal{L}\left(A_{\ell}, M_{\ell}(u)\right), \sum_{\ell=0}^{d} A_{\ell} \mathcal{U}\left(A_{\ell}, M_{\ell}(u)\right)\right] .
$$

Here, for every $0<\underline{x}<x<\bar{x}$, being $\underline{x}, \bar{x}$ rational numbers, we have

$$
\mathcal{L}(A, x)=\left\{\begin{array}{ll}
\underline{x} & \text { if } A>0, \\
0 & \text { if } A=0, \\
\bar{x} & \text { if } A<0,
\end{array} \quad \text { and } \quad \mathcal{U}(A, x)= \begin{cases}\bar{x} & \text { if } A>0, \\
0 & \text { if } A=0, \\
\underline{x} & \text { if } A<0 .\end{cases}\right.
$$

We notice that $h$ is chosen in such way that the maximal and minimal values of each monomial are taken in the vertices of $[-h, h]^{n}$.

\section{ACKNOWLEDGEMENTS}

This work has received funding from the Ministerio de Economía, Industria y Competitividad - Agencia Estatal de Investigación (FEDER Grant MTM2016-77278P), the Agència de Gestió d'Ajuts Universitaris i de Recerca (Grant 2017 SGR 1617), the Brazilian agencies FAPESP (Grants 2013/24541-0 and 2017/03352-6), CAPES (Grant PROCAD 88881.068462/2014-01), CNPq (Grant 308006/2015-1), FAPEG (29199/2018), and FAPEG/CNPq (Grant PRONEX 2017-10267000508), and the European Union's Horizon 2020 research and innovation programme (Grant Dynamics-H2020-MSCA-RISE-2017-777911).

\section{REFERENCES}

[1] V. Acary, O. Bonnefon, and B. Brogliato. Nonsmooth modeling and simulation for switched circuits, volume 69 of Lecture Notes in Electrical Engineering. Springer, Dordrecht, 2011.

[2] A. A. Andronov, E. A. Leontovich, I. I. Gordon, and A. G. Mă̌er. Theory of bifurcations of dynamic systems on a plane. Halsted Press [A division of John Wiley \& Sons], New YorkToronto, Ont., 1973.

[3] A. A. Andronov, A. A. Vitt, and S. E. Khalkin. Theory of oscillators. Dover Publications, Inc., New York, 1987. Translated from the Russian by F. Immirzi, Reprint of the 1966 translation.

[4] M. Apri, N. Banagaaya, B. van den J. B, R. Brussee, D. Bourne, S. Fatima, F. Irzal, J. Rademacher, B. Rink, F. Veerman, S. Verpoort, and J. Hulshof. Analysis of a model for ship maneuvering. In Proceedings of the 79 th European Study Group Mathematics with Industry, pages 83-116. Vrije Univ. Amst., 2011.

[5] C. A. Buzzi, T. de Carvalho, and M. A. Teixeira. Birth of limit cycles bifurcating from a nonsmooth center. J. Math. Pures Appl. (9), 102(1):36-47, 2014.

[6] C. A. Buzzi, C. Pessoa, and J. Torregrosa. Piecewise linear perturbations of a linear center. Discrete Contin. Dyn. Syst., 33(9):3915-3936, 2013.

[7] P. T. Cardin and J. Torregrosa. Limit cycles in planar piecewise linear differential systems with nonregular separation line. Phys. D, 337:67-82, 2016.

[8] C. Chicone and M. Jacobs. Bifurcation of critical periods for plane vector fields. Trans. Amer. Math. Soc., 312(2):433-486, 1989. 
[9] C. Christopher. Estimating limit cycle bifurcations from centers. In Differential equations with symbolic computation, Trends Math., pages 23-35. Birkhäuser, Basel, 2005.

[10] B. Coll, R. Prohens, and A. Gasull. The center problem for discontinuous Liénard differential equation. Internat. J. Bifur. Chaos Appl. Sci. Engrg., 9(9):1751-1761, 1999. Discrete dynamical systems.

[11] M. di Bernardo, C. J. Budd, A. R. Champneys, and P. Kowalczyk. Piecewise-smooth dynamical systems. Theory and applications, volume 163 of Applied Mathematical Sciences. SpringerVerlag London, Ltd., London, 2008.

[12] F. Dumortier, J. Llibre, and J. C. Artés. Qualitative theory of planar differential systems. Universitext. Springer-Verlag, Berlin, 2006.

[13] J. Fan, S. Xue, and G. Chen. On discontinuous dynamics of a periodically forced double-belt friction oscillator. Chaos Solitons Fractals, 109:280-302, 2018.

[14] A. F. Filippov. Differential equations with discontinuous righthand sides, volume 18 of Mathematics and its Applications (Soviet Series). Kluwer Academic Publishers Group, Dordrecht, 1988.

[15] E. Freire, E. Ponce, F. Rodrigo, and F. Torres. Bifurcation sets of continuous piecewise linear systems with two zones. Internat. J. Bifur. Chaos Appl. Sci. Engrg., 28(11):2073-2097, 1998.

[16] E. Freire, E. Ponce, and F. Torres. The discontinuous matching of two planar linear foci can have three nested crossing limit cycles. Publ. Mat., 58(suppl.):221-253, 2014.

[17] E. Freire, E. Ponce, and F. Torres. A general mechanism to generate three limit cycles in planar Filippov systems with two zones. Nonlinear Dynam., 78(1):251-263, 2014.

[18] M. Han. Liapunov constants and Hopf cyclicity of Liénard systems. Ann. Differential Equations, 15(2):113-126, 1999.

[19] S. Huan and X.-S. Yang. Generalized Hopf bifurcation emerged from a corner in general planar piecewise smooth systems. Nonlinear Anal., 75(17):6260-6274, 2012.

[20] W. Kulpa. The Poincaré-Miranda theorem. Amer. Math. Monthly, 104(6):545-550, 1997.

[21] J. Llibre and E. Ponce. Three nested limit cycles in discontinuous piecewise linear differential systems with two zones. Dyn. Contin. Discrete Impuls. Syst. Ser. B Appl. Algorithms, 19(3):325-335, 2012.

[22] J. C. Lucero and C. A. Gajo. Oscillation region of a piecewise-smooth model of the vocal folds. Commun. Math. Sci., 4(2):453-469, 2006.

[23] R. Lum and L. O. Chua. Global properties of continuous piecewise-linear vector fields. Part I: Simplest case in $R^{2}, 1990$. Memorandum UCB/ERL M90/22, University of California at Berkeley.

[24] J. C. Medrado and J. Torregrosa. Uniqueness of limit cycles for sewing planar piecewise linear systems. J. Math. Anal. Appl., 431(1):529-544, 2015.

[25] I. Niven. Irrational numbers. The Carus Mathematical Monographs, No. 11. The Mathematical Association of America. Distributed by John Wiley and Sons, Inc., New York, N.Y., 1956.

[26] V. G. Romanovski and D. S. Shafer. The center and cyclicity problems: a computational algebra approach. Birkhäuser Boston, Inc., Boston, MA, 2009.

[27] R. Roussarie. Bifurcations of planar vector fields and Hilbert's sixteenth problem. Modern Birkhäuser Classics. Birkhäuser/Springer, Basel, 1998.

[28] Y. Zou and T. Küpper. Generalized Hopf bifurcation emanated from a corner for piecewise smooth planar systems. Nonlinear Anal., 62(1):1-17, 2005.

Departamento de Matemática, Universidade Estadual Paulista, 15054-000 São José Do Rio PREto, Brazil

Email address: claudio.buzzi@unesp.br

Instituto de Matemática e Estatística, Universidade Federal de Goiás, 74001-970 GoiÂnia, Goiás, BraziL

Email address: medrado@ufg.br

Departament de Matemàtiques, Universitat Autònoma de Barcelona, 08193 BelLATERra, BARCElona, Spain

Email address: torre@mat.uab.cat 